\title{
Research on the Novel Class Design in Meteorological Teaching
}

\author{
Xu Guanlei, Shao Limin \\ Meteorological Teaching Room of Ocean Department \\ Dalian Navy Academy \\ Dalian, China \\ xgl_86@163.com
}

\author{
Liu Yonglu, Lang Fengwang \\ Meteorological Teaching Room of Ocean Department \\ Dalian Navy Academy \\ Dalian, China \\ xgl_86@sohu.com
}

\begin{abstract}
Meteorology is the key course in many specialties in some colleges and universities. In this field, we know that the wind is one of the most important weather elements. In addition, the types, the production mechanism and features of wind are very important for the sailors on the sea in the aspect of navigation safety. This paper proposes a novel class design in meteorological teaching for studying the wind in the aspects of the production of wind, the types of wind, the features of different winds and the applications as well. The novel design is defined as follows: the study is introduced by the real-world question so that the students will pay more attention in classes, then a framework is given so that all the content belongs to a whole body and the students will have more interests in the study. Finally, experiments are given to show our novel class design's efficiency.
\end{abstract}

Keywords-meteorology; wind; meteorological teaching; cyclone ; anticyclone

\section{INTRODUCTION}

It is very well known that the interest is the best teacher in the teaching and studying [1-2]. In the classroom, one of the most important things is that the college students are very interested in the content introduced by the teachers. As a college student, if the thing he is studying is very interesting for him, then he will pay more attentions on it. The result is that he will learn more on this thing. Interest will make the student persistent and willing to put in the effort. In order to let the students be interested in the content taught by the teachers is the teachers' research work in their classroom teaching. It is very important for the difficult courses such as synoptic meteorology or meteorological teaching. So, in order to let the courses of synoptic meteorology or meteorological teaching interesting, our first work will be to make a novel class design in classroom.

Meteorology is the key course in many specialties in our academy [3-6]. It is also the elementary content and basic framework in our students' study. In this field, the wind is one of the most important weather elements. The types, the production mechanism and features of wind are very important for the sailors on the sea in the aspect of navigation safety. This paper mainly involves the production of wind, the types of wind, the features of different winds and the applications in the meteorological teaching class.

Therefore, this paper will propose a novel class design in meteorological teaching for studying the wind in the aspects of the production of wind, the types of wind, the features of different winds and the applications. The novel design is defined as follows: the study is introduced by the real-world question so that the students will pay more attention in classes, then a framework is given so that all the content belongs to a whole body and the students will have more interests in the study. In the end of our paper, some experiments will be given to show our novel class design's efficiency.

\section{TeAching Design}

We will adopt the scheme of question-guiding and illumination, and at the same time we will take the traditional typhoon as the real-world example that will be our question guiding the teaching. The different action forces will be our main masterstroke, also the cyclone and the anticyclone are employed to introduce the wind direction and wind strength and so on [3-6]. Gradually, we will introduce the students to think about the real-world wind and its features on the sea so that the students can understand the real essence of the wind to make their navigation better and safer [1]. The details are as follows:

\section{A. Teaching Object}

The students should mainly master the wind types, the production mechanism and the features including the difference and sameness of the wind between the cyclone and the anticyclone. The main task is to let the students know the exact drawing of the real wind in the meteorological fax maps. Letting the students think the real-world questions carefully on their own is one of our main tasks. At the same time, letting the students raise the real-world questions on their own and resolve them is also one of our main tasks.

\section{B. Teaching Task}

The analysis of students before teaching: up till now, the students have studied the elementary theory and the action forces of air, and they have mastered the cases of the action forces of air. On the other hand, they have studied the meteorological fax maps before this study. However, since their former study is not deep, they cannot understand these

This work is sponsored by the NSFCs (61471412, 61771020). 
knowledge points well. We must teach them the detailed drawing of wind direction and wind power in the form of theoretical system in the real meteorological fax maps so that in the later section of the class they can follow the teachers' ideas.

The analysis of contents before teaching: these contents seem to be very complicated and difficult, however, in fact if the idea of them is clear, then these contents will be very easy. Here one question is our guiding, following which the production of wind, the types of wind, the features of different winds and the applications are introduced in great details. This will accord to the original question raised by our guiding question.

\section{Teaching Scheme}

The teaching will mainly focus on the theoretical explaining combined with illumination and exchanges by the form of blackboard and ppt. The class teaching will begin from an example or question (i.e., we will mainly give the students the real-world meteorological fax maps and ask them some questions), then the questions will be raised. The questions are twofold: the first one is why the wind power far away from the center of the anticyclone is big and instead the wind around the center of the cyclone is big? The second one is why the max wind power grade in the anticyclone is always less than 12 and the max wind power grade in the cyclone is often more than 12 ? In the follows, we will take the different action forces as our main masterstroke to explain the production of wind, the types of wind, the features of different winds and the applications in great details combined with the difference between the two weather systems via comparisons. Finally, our explaining and the detailed teaching will answer the above question raised by us at the beginning of the class. Thus the students will master these knowledge points and they will have more interests in these problems.(see Fig.1 and Fig.2)

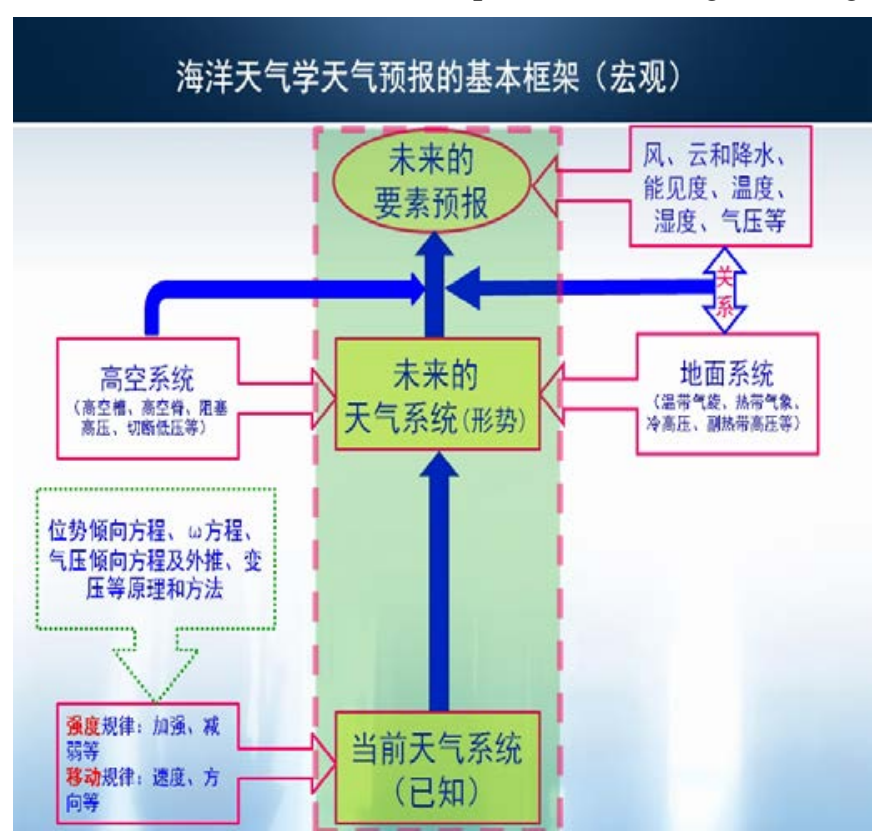

Fig. 1. The total framework in teaching.

\section{The Design of Teaching}

First step, we will review the last classes and ask the students about the main knowledge points. At the same time, in the ppt we will give the equations about these forces enforced on wind by the follows:

$$
\begin{aligned}
& G=-\frac{1}{\rho} \nabla P=-\frac{1}{\rho}\left\{\begin{array}{l}
\partial P / \partial x \\
\partial P / \partial \mathrm{y} \\
\partial P / \partial \mathrm{z}
\end{array}\right\}, \\
& F=-K \cdot V, \\
& A=\left\{A_{x}, A_{y}, A_{z}\right\}^{T}=\left\{\begin{array}{l}
2 \Omega v \sin \varphi-2 \Omega w \cos \varphi \\
-2 \Omega u \sin \varphi \\
2 \Omega u \cos \varphi
\end{array}\right\}, \\
& C=\frac{V^{2}}{R} .
\end{aligned}
$$

Note that these forces belong to the two types that every student must know well.

The second step, we give a real-world meteorological fax map shown in Fig. 1 in the ppt to ask the students the questions. Note that in Fig.1, there are some important sentences such as “T 1330 HAIYAN...GUST 165KT...EXPECTED GUST $175 \mathrm{KT} . .$. " and so on. These sentences are our key points.

From the map, we will raise the two questions: the first one is why the wind power far away from the center of the anticyclone is big and instead the wind around the center of the cyclone is big? The second one is why the max wind power grade in the anticyclone is always less than 12 and the max wind power grade in the cyclone is often more than 12 ?

Third step, we will give the masterstroke as follows:

$$
\begin{aligned}
& G+A=0, \\
& G+A+C=0, \\
& G+A+C+F=0 .
\end{aligned}
$$

Fourth step, we will introduce the first type of wind and give the following equations combined with their concept, features and wind directions and so on.

$$
\begin{aligned}
& \left\{\begin{array}{l}
0=-\frac{1}{\rho} \frac{\partial P}{\partial x}+f v \\
0=-\frac{1}{\rho} \frac{\partial P}{\partial y}-f u
\end{array}\right. \\
& \left\{\begin{array}{l}
u_{g}=-\frac{1}{f \rho} \frac{\partial P}{\partial y} \\
v_{g}=\frac{1}{f \rho} \frac{\partial P}{\partial x}
\end{array}\right.
\end{aligned}
$$




$$
\vec{V}_{g}=-\frac{1}{f \rho} \nabla_{h} P \times \vec{k}
$$

Fifth step, we will introduce the second type of wind and give the following equations combined with their concept, features and wind directions and so on.

$$
\begin{aligned}
& \left\{\begin{array}{l}
0=-\frac{1}{\rho} \frac{\partial P}{\partial s} \\
\frac{v^{2}}{R}=-\frac{1}{\rho} \frac{\partial P}{\partial n}-f v
\end{array}\right. \\
& \left\{\begin{array}{l}
-\frac{1}{\rho} \frac{\partial P}{\partial s}=0 \\
-\frac{1}{\rho} \frac{\partial P}{\partial n}-f v-\frac{v^{2}}{R}=0 \quad \mathrm{n}
\end{array}\right. \\
& v_{f}=-\frac{R_{T}}{2} f \pm \frac{R_{T}}{2} \sqrt{f^{2}-\frac{4}{R_{T} \rho} \frac{\partial P}{\partial n}} .
\end{aligned}
$$

Sixth step, we will introduce the real wind near the ground combined with their concept, features and wind directions and so on.

Seventh step, we conclude our class.

Eighth step, we will give some homework to the students.

In a word, through the above eight steps we can explain these knowledge points very well.

\section{EXPERIMENTS AND DISCUSSION}

The design of our class has the following features:

(1)The professional meteorological features are outstanding. This design not only has the theoretical content, but also has the applications in the real world. So the professional meteorological feature is very clear.

(2)The design of the class is suitable. The two questions are raised by us, the first one is the reason the wind power far away from the center of the anticyclone is big and instead the wind around the center of the cyclone is big; And the second one is reason the max wind power grade in the anticyclone is always less than 12 and the max wind power grade in the cyclone is often more than 12 . These questions will make the students have more interests in the studying the classes.

(3)The content is huge. In the class, a lot of information is introduced to the students including the old, the new, the theory, the application and so on.

(4)The realistic importance is large. Finally, our focus is placed on the applications of the weather forecasting, therefore, the realistic importance is large.

At the same time, we use our class design to test our students for the efficiency of studying these contents. We find that if the design is given to the students, then they will understand these points better. The average score is improved by $9.4 \%$ (see Table I for the details). So it is very clear that our design is very useful.

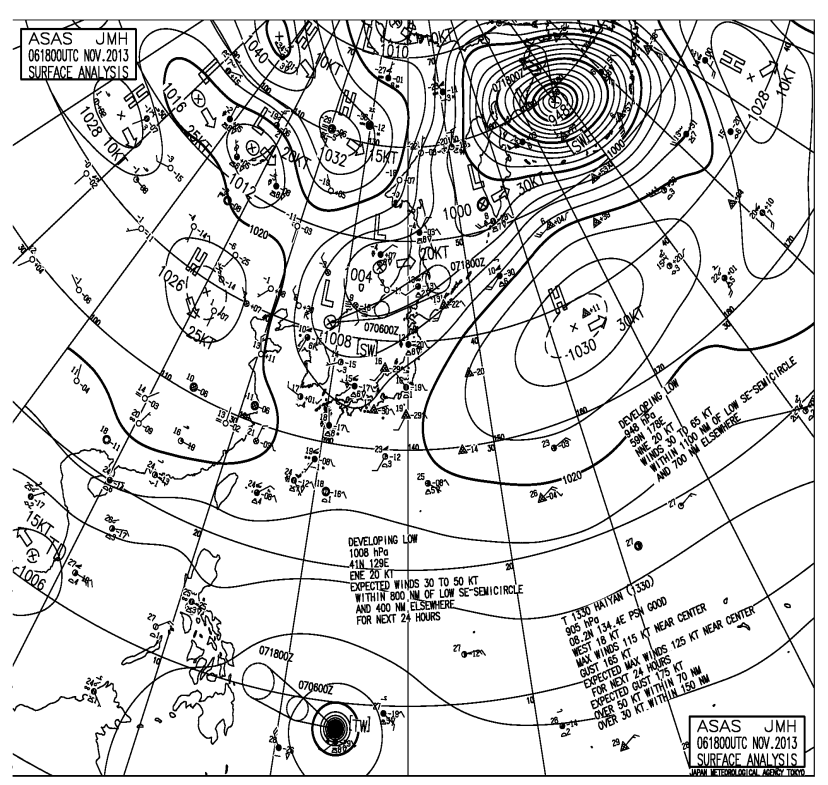

(a)

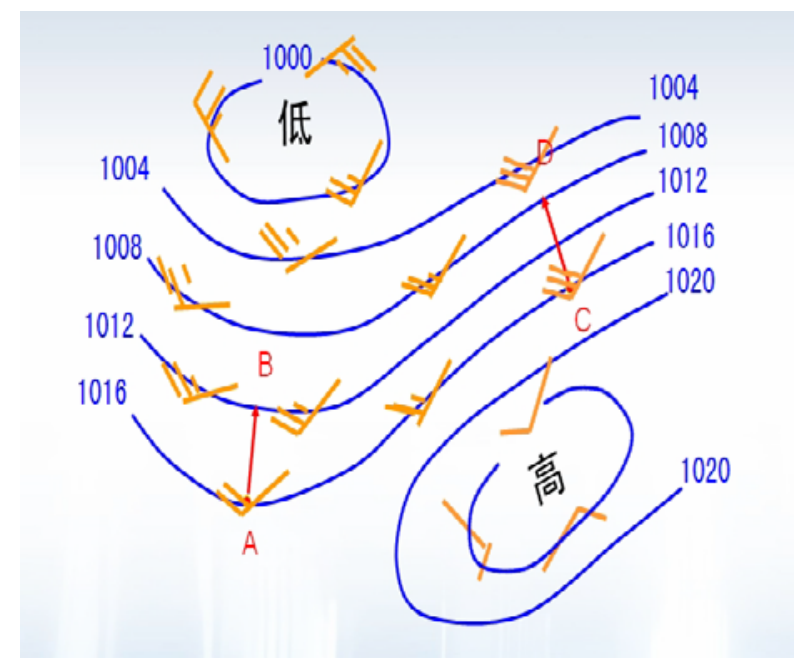

(b)

Fig. 2. An example of a real-world meteorological fax.

TABLE I. THE AVERAGE SCORE COMPARISON

\begin{tabular}{|c|c|c|c|c|}
\hline \multirow{2}{*}{ Terms } & \multicolumn{4}{|c|}{ Comparisons } \\
\cline { 2 - 5 } & $\begin{array}{c}\text { Without } \\
\text { Design } \\
\text { number }\end{array}$ & $\begin{array}{c}\text { Average } \\
\text { Scores }\end{array}$ & $\begin{array}{c}\text { With } \\
\text { Design } \\
\text { number }\end{array}$ & $\begin{array}{c}\text { Average } \\
\text { Scores }\end{array}$ \\
\hline Grade 1 & 100 & 78.2 & 88 & $\mathbf{8 6 . 5}$ \\
\hline Grade 2 & 95 & 76.3 & 89 & $\mathbf{8 3 . 9}$ \\
\hline Grade 3 & 102 & 77.9 & 98 & $\mathbf{8 5 . 3}$ \\
\hline Grade 4 & 57 & 79.0 & 64 & $\mathbf{8 4 . 4}$ \\
\hline
\end{tabular}




\section{CONCLUSIONS}

It is very well known that the interest is the best teacher in the teaching/studying. In order to let the courses of meteorological teaching interesting, the first work is to make a novel class design in classroom. As a college student, if the knowledge he is studying is very interesting for him, then he will pay more attentions on the content. The result is that he will learn more on this knowledge. Interests will make the students persistent and willing to put in the effort. This paper gives a novel class design in the meteorological teaching for studying the wind in the production of wind, the types of wind, the features of different winds and the applications. Meteorology is the key course in many colleges and universities. It is also the elementary content and basic frame in the students' studying. In the field, the wind is one of the most important weather elements for weather forecasting. The types, the production mechanism and features of wind are very important for the sailors on the sea in the aspect of navigation safety. The novel design is as follows: the study is introduced by the real-world question so that the students will pay more attention in classes, then a framework is given so that all the content belongs to a whole body and the students will have more interests in the study. At last, some experiments are given to show the proposed novel class design's efficiency, i.e., if the design is given to the students, then they will understand these points better. The average score is improved by $9.4 \%$. Future work will include more analysis via the tools of image processing [7-11].

\section{ACKNOWLEDGMENT}

We will thank the anonymous college students in our classroom for their cooperation with our experiments.

\section{REFERENCES}

[1] Xu Guanlei, Shao Limin, Liu Yonglu, Lang Fengwang. The Content Classification via Bayesian Model in Meteorological Teaching [C]. Advances in Social Science, Education and Humanities Research (ASSEHR), 2016, volume 75:11-14.

[2] Yang Jinyan, Xu Guanlei, Li Yu, Zou Qiyang. College Student Classification in Swimming Teaching and Training[C]. Advances in Social Science, Education and Humanities Research (ASSEHR), 2016, volume 75:7-10.

[3] https://wenku.baidu.com/view/d2c91a08cc22bcd126ff0caf.html.

[4] Walsh J E, Chapman W L. Short-Term Climatic Variability of the Arctic[J]. J. Climate, 1990, 3, 237-250.

[5] Fan J . Design-adaptive nonparametric regression . Journal of American Statistical Association, 1992, $87: 998 \sim 1004$.

[6] McCann D W. A neural network short-term forecast of significant thunderstorms $[\mathrm{J}]$. Weather and Forecasting, 1992, 26(7) : 525 534.

[7] Xu Guanlei, Wang Xiaotong, Zhou Lijia, Xu Xiaogang. Image Decomposition and Texture Analysis via Combined Bi-Dimensional Bedrosian's Principles, IET Image Processing, 2018(preprinted).

[8] Xu Guanlei, Wang Xiaotong, Xu Xiaogang, Zhou Lijia. Entropic Inequalities on Sparse Representation[J]. IET Signal Processing, 2016,10(4) : :413-421.

[9] Xu Guanlei, Wang Xiaotong, Zhou Lijia, Xu Xiaogang.New Inequalities on Sparse Representation in Pairs of Bases [J]. IET Signal Processing, 2013, 7(8), pp.674-683.

[10] Xu Guanlei, Zhou Lijia, Wang Xiaotong, Xu Xiaogang. Assisted Signals Based Mode Decomposition, International Conference on Image, Vision and Computing, 2017 :868-874.

[11] Xu Guanlei, Wang Xiaotong, Zhou Lijia, Xu Xiaogang. Image Decomposition and Texture Analysis via Combined Bi-Dimensional Bedrosian's Principles, IET Image Processing, 2018, 12 (2): 262-273. 